\title{
The Upgrade of the ATLAS Level-1 Central Trigger Processor
}

\author{
TWEPP 2012 \\ Oxford, 18. September 2012
}

G. Anders, D. Berge, H. Bertelsen, M. Dam, E. Dobson, N. Ellis, P. Farthouat, C. Gabaldon Ruiz, M, Ghibaudi, B. Gorini, S. Haas, M. Kaneda, A. Messina, T. Pauly, R. Pottgen, R. Spiwoks,

T. Wengler, S. Maettig, M. Stockton, S. Xella 


\section{Outline}

- Current ATLAS Level-1 central trigger architecture

- Phase-0 trigger upgrade (2014)

- Central Trigger Processor (CTP) functionality and architecture

- Motivation for CTP upgrade and specification

- New CTPCORE module design

- Implementation

- Firmware

- New CTPOUT module \& backplane design

- Summary 


\section{ATLAS Level-1 Trigger System}

- Process reduced granularity information from calorimeter and muon detectors

- Trigger decision based on object multiplicities at different thresholds

- Generate Level-1 Accept (L1A) and send via Timing, Trigger and Control (TTC) distribution to detector front-ends to initiate readout

- Identify regions-of-interest (Rol) to seed the LVL2 trigger

- Synchronous, pipelined processing system operating at the bunch crossing $(\mathrm{BC})$ rate of $40 \mathrm{MHz}$

- Maximum round-trip latency: 2.5 us

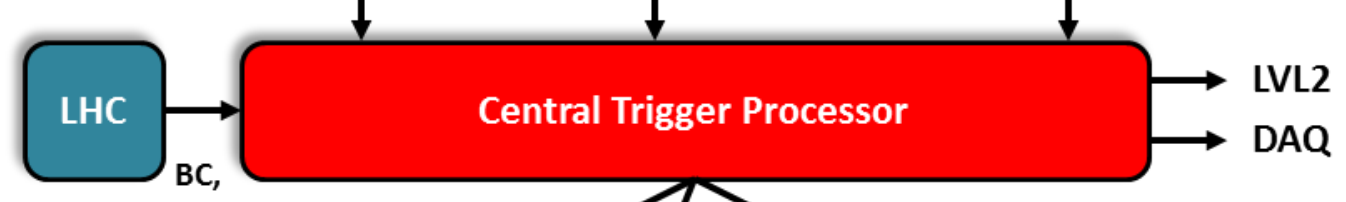

- Data stored in on-detector pipelines

- Maximum trigger rate: $75 \mathrm{kHz}$

- Custom built electronics 


\section{Phase-0 Trigger Upgrade (2014)}

- Calorimeter trigger

- Replace PreProcessor multi-chip modules (MCMs)

- Replace merger modules (CMX)

- Send L1Calo Rols to topological processor

- Topological trigger (new)

- Topological processor with calorimeter and subset of muon Rol input

- Improve multi-object selection for increasing luminosity

- Central trigger (upgraded)

- New CTP modules to add more inputs and extend number of menu items

- Latency and trigger rate limits remain unchanged until Phase-II

- 2.5 us round-trip latency

- 20 BC headroom

- $75 \mathrm{kHz}$ (upgradeable to $100 \mathrm{kHz}$ )

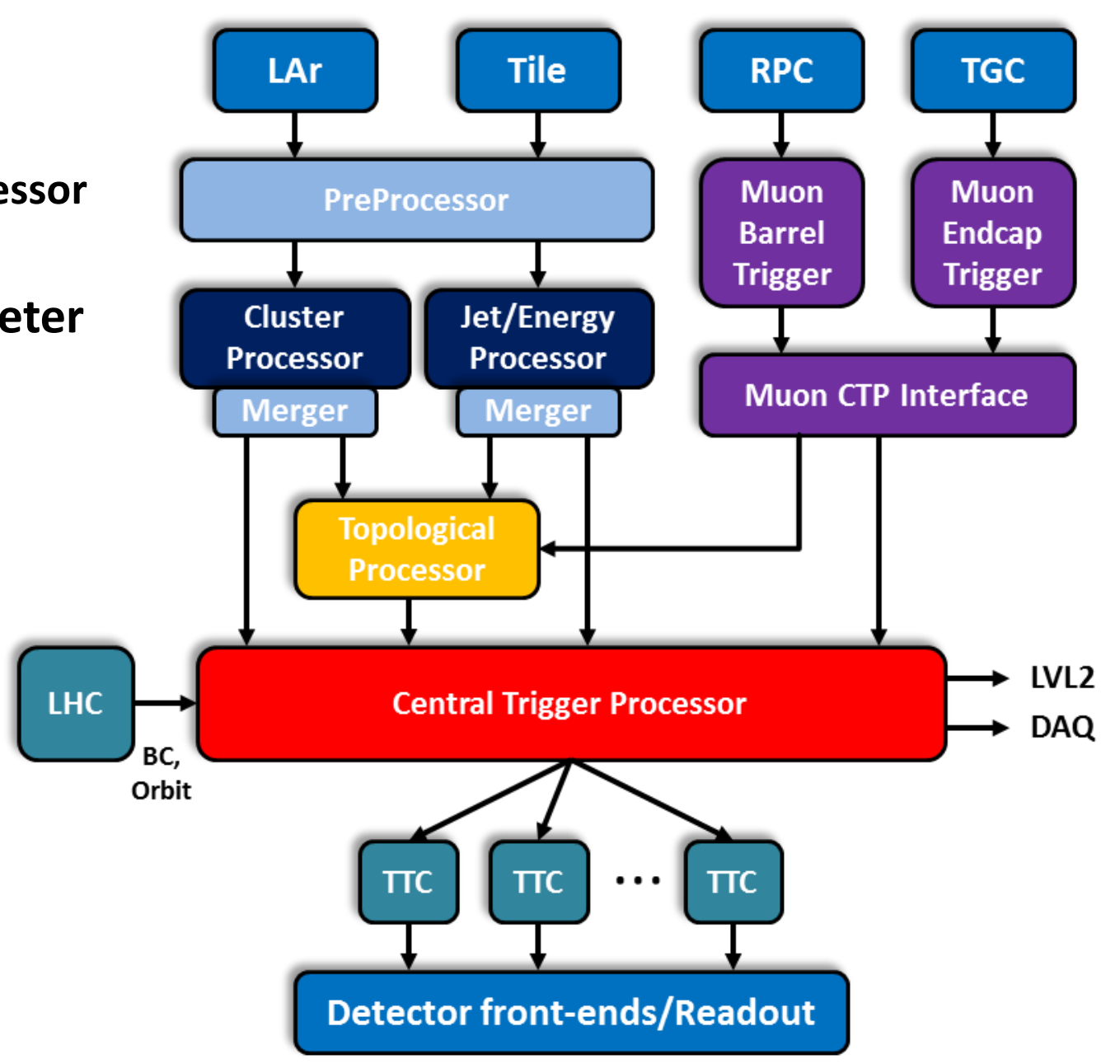

- Limited by readout 


\section{CTP Functionality}

- Level-1 trigger decision (L1A generation)

- Low latency: 4 BC

- Combine triggers from calorimeter \& muon trigger processors and forward detectors

- Trigger item formation

- Flexible logical combinations of trigger inputs as defined in the trigger menu

- Bunch group masking

- Masking of triggers as a function of LHC bunch structure

- Trigger item pre-scaling

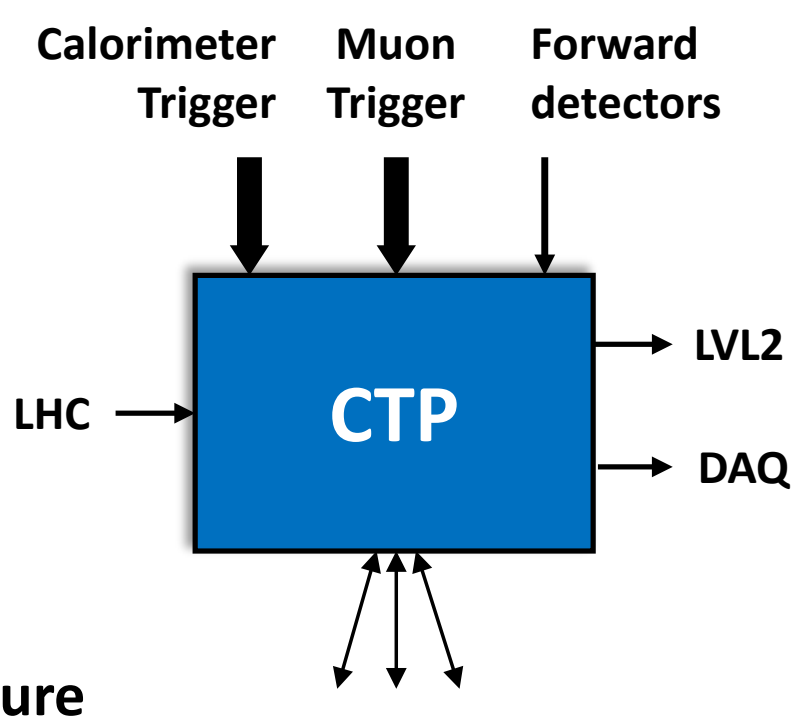

TTC Partitions

- Preventive dead-time generation to protect detector front-ends

- Simple (fixed number of bunches) and complex dead-time (leaky bucket algorithm)

- Busy from readout (busy tree)

- Receive timing signals (BC, ORBIT) from the LHC

- Fan-out trigger and timing signals to TTC partitions, receive busy

- Send Rol summary information to LVL2 and event data to DAQ

- Online monitoring of trigger, dead-time and busy

- Provides essential information for luminosity and background monitoring 


\section{CTP Architecture}

- 9U VME chassis with 11 modules

- CTPMI - Machine interface

- Receives timing signals from LHC

- 3 x CTPIN - Input module

- Receives, synchronizes and aligns trigger input signals

- CTPMON - Monitoring module

- Performs bunch-by-bunch trigger monitoring
- CTPCORE - Core module

- Generates Level-1 Accept (L1A)

- Sends event summary information to LVL2 \& DAQ

- 4 x CTPOUT - Output module

- Send timing signals to LTPs

- 3 custom backplanes

- Trigger \& timing (COM), Trigger inputs (PIT) \& calibration requests (CAL)

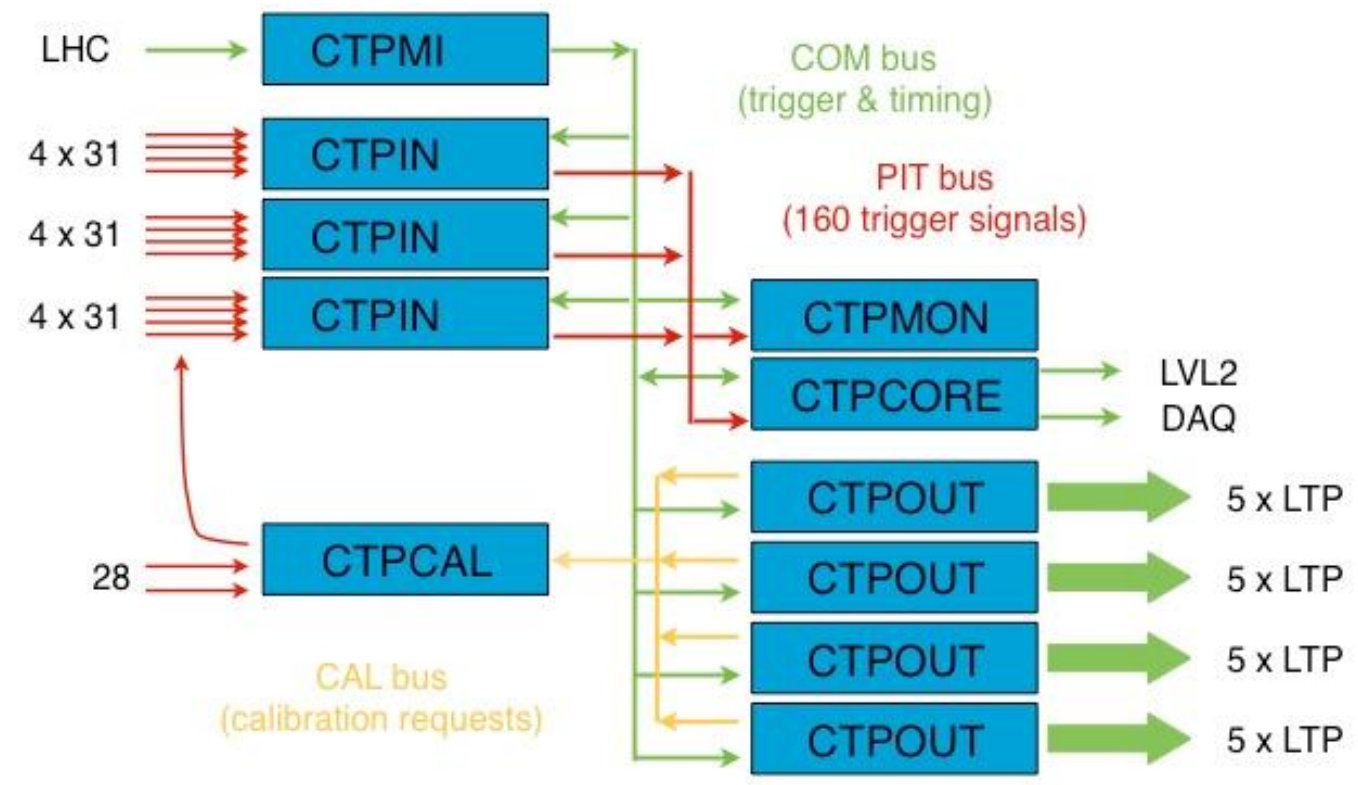




\section{CTP Resource Utilisation}

- CTP in ATLAS is working very reliably

- No spare capacity for some of the resources

- CTP utilization from 2012 trigger menu:

\begin{tabular}{|l|r|r|}
\hline CTPIN input cables (partially used) & Used & Available \\
\hline CTPIN input signals & 2 & 12 \\
\hline CTPIN integrating monitoring counters & 212 & 372 \\
\hline PIT bus lines & 138 & 768 \\
\hline CTPCORE trigger items & 160 & 160 \\
\hline CTPCORE bunch group masks & 241 & 256 \\
\hline CTPCORE maximum number of AND terms & 8 & 8 \\
\hline CTPCORE maximum number of bits in OR terms & 6 & 256 \\
\hline CTPCORE per-bunch trigger item counters & 6 & 12 \\
\hline CTPOUT cables to TTC partitions & 12 & 12 \\
\hline CTPMON per-bunch monitoring counters & 20 & 20 \\
\hline
\end{tabular}




\section{Motivation for Upgrade}

- Primary motivation: remove CTP resource limitations

- Increase the number of trigger inputs

- Increase the number of trigger items (combinations)

- Additional features

- Allow partitioning of L1A generation for detector commissioning

- Improved bunch group masking and per-bunch trigger item monitoring

- Low latency direct electrical inputs from Topological processor to CTPCORE

- Option to use optical inputs to connect to new/upgraded sub-systems (latency budget permitting)

- CTP upgrade requires complete redesign of several modules

- CTPCORE

- CTPOUT

- COM backplane

- Next major CTP upgrade foreseen only for Phase-II

- Level-1 trigger architecture changes -> 2 hardware trigger levels (L0/L1)

- Latency budget increases 


\section{Phase-0 CTP Specifications}

- 320 trigger inputs on PIT bus backplane (now 160)

- Using double data rate signalling on the existing PIT bus backplane

- Previous study has shown proof of principle

- Latency penalty of 2 BC

- 512 trigger items (now 256)

- 4 trigger partitions (now 1)

- Common trigger menu

- Each L1A partition has selection of trigger items (mask), and it's own dead-time handling

- Only primary L1A partition (i.e. "physics" partition) provides LVL2 and DAQ readout

- Secondary L1A partitions used for detector commissioning, calibration, etc.

- 16 bunch groups (now 8)

- After forming the trigger items (now part of trigger item)

- 256 per-bunch counters for trigger item monitoring (now 12)

- 64 electrical LVDS inputs for L1Topo trigger signals to CTPCORE

- 12 serial optical inputs using ribbon-fiber receiver

- Overall latency target 6-7 BC 


\section{CTPCORE++ Module Design}

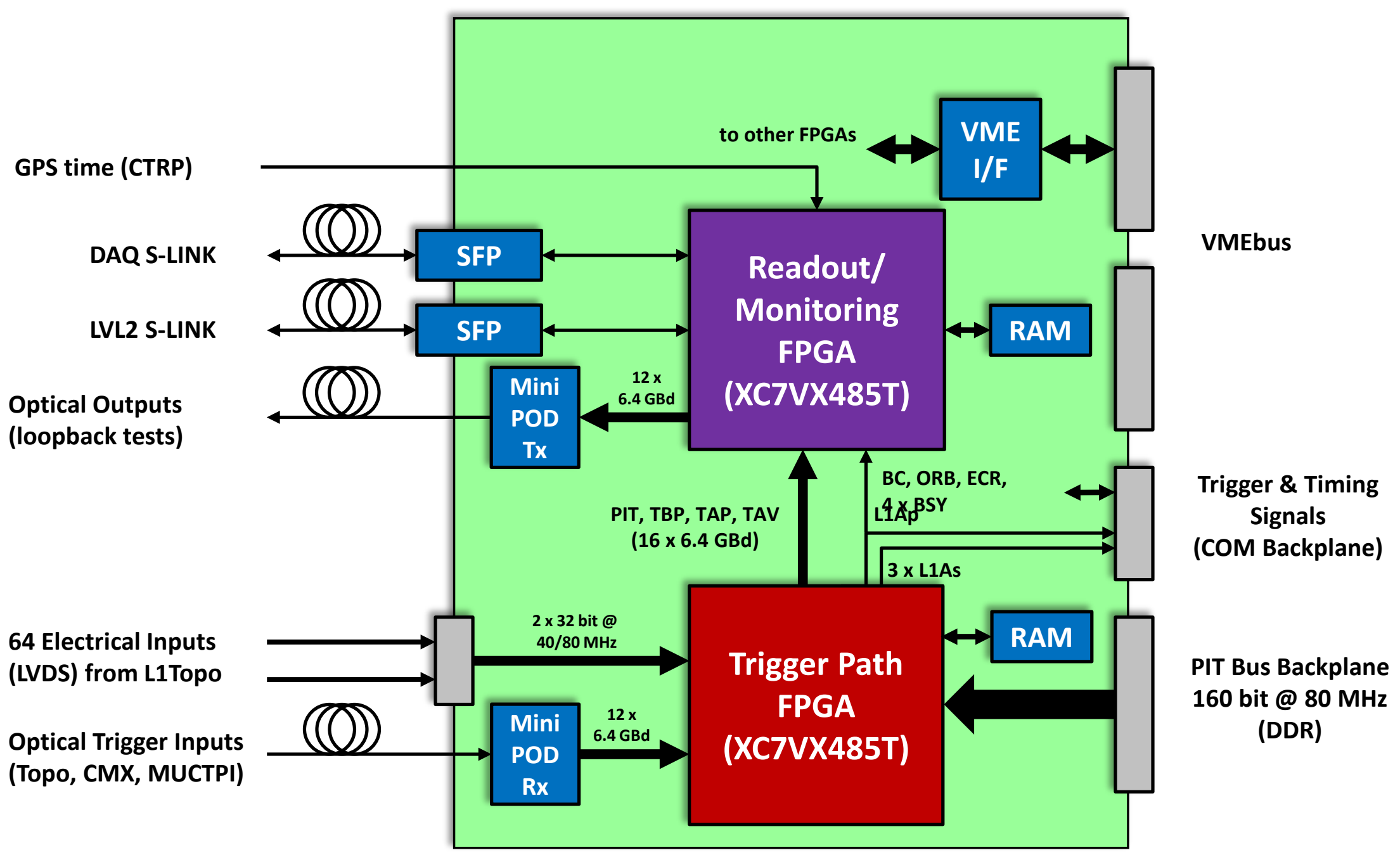

- Schematics design in progress

- Prototype expected in Q1'2013 


\section{Implementation (1)}

- Two Virtex-7 FPGAs (XC7VX485T, BGA1157)

- $600 \mathrm{l} / 0$ pins, 20 multi-gigabit transceivers (GTX), 300k LUTs (6-input), 600k flip-flops, 1030 RAM blocks (36 kbit)

- Possibility to migrate to smaller or larger densities in same package for production modules

- Need to send 2000 bit/BC from trigger path FPGA to readout/monitoring FPGA ( $80 \mathrm{Gbit} / \mathrm{s}$ )

- Requires 16 serial links @ 6.4 Gbaud

- 8B10B encoding, $120 \mathrm{bit} / \mathrm{link} / \mathrm{BC}$

- DDR3 playback and diagnostics memories

- Inject test patterns \& store results

- $2000 \mathrm{bits} / \mathrm{BC}=>80 \mathrm{Gbit} / \mathrm{s}$

- Two DDR3 SODIMM modules

- Requires 60\% transfer efficiency @ 1066 Mb/s

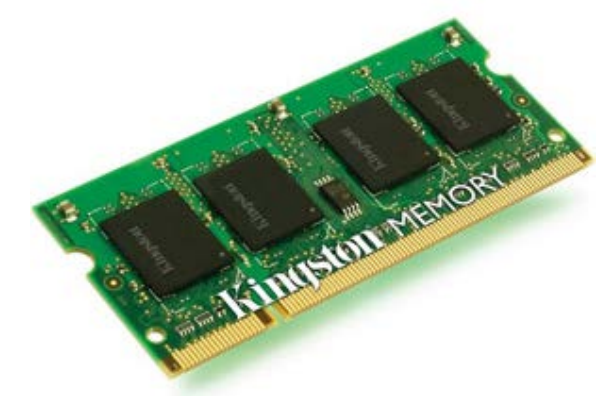




\section{Implementation (2)}

- Optical inputs

- Avago MiniPOD parallel ribbon fibre transmitters/receivers

- High density $(\sim 2 \times 2 \mathrm{~cm})$, can be placed close to FPGA

- Eases signal integrity issues (short traces)

- Link speed (conservative): 6.4 GBd, 8B10B coded

- Up to $128 \mathrm{bit} / \mathrm{BC} /$ fiber $\Rightarrow>$ more than enough

- Using fewer bits than 128 per link can somewhat reduce link latency

- Latency penalty $3 \mathrm{BC}$

- Initially use electrical inputs, migrate to optical when required

- Design challenges

- Signal integrity of high-speed serial links

- Power supply distribution is complex

- Many low-voltage (1.0V, 1.2V, 1.8V, 2.5V) digital supplies for FPGAs

- Separate low-noise supplies for transceivers (1.0V, 1.2V, 1.8V)

- Virtex-7 I/O only support signal levels up to $1.8 \mathrm{~V}$ : level translation required

- PIT bus (2.5V SSTL) -> CPLD with different I/O bank voltages

- VME -> small FPGA with $3.3 \mathrm{~V}$ and $1.8 \mathrm{~V} \mathrm{I} / \mathrm{O}$ banks

- LVDS receivers -> NMOS pass transistors for $3.3 \mathrm{~V}$ to $1.8 \mathrm{~V}$ translation 


\section{CTPCORE++ Trigger Path}

- Trigger path implemented in one FPGA to reduce latency

- Initial trigger path firmware implementation shows a latency of $\sim 2$ BC

- Dominated by routing delays

- Second FPGA for non-latency critical functions (readout \& monitoring)

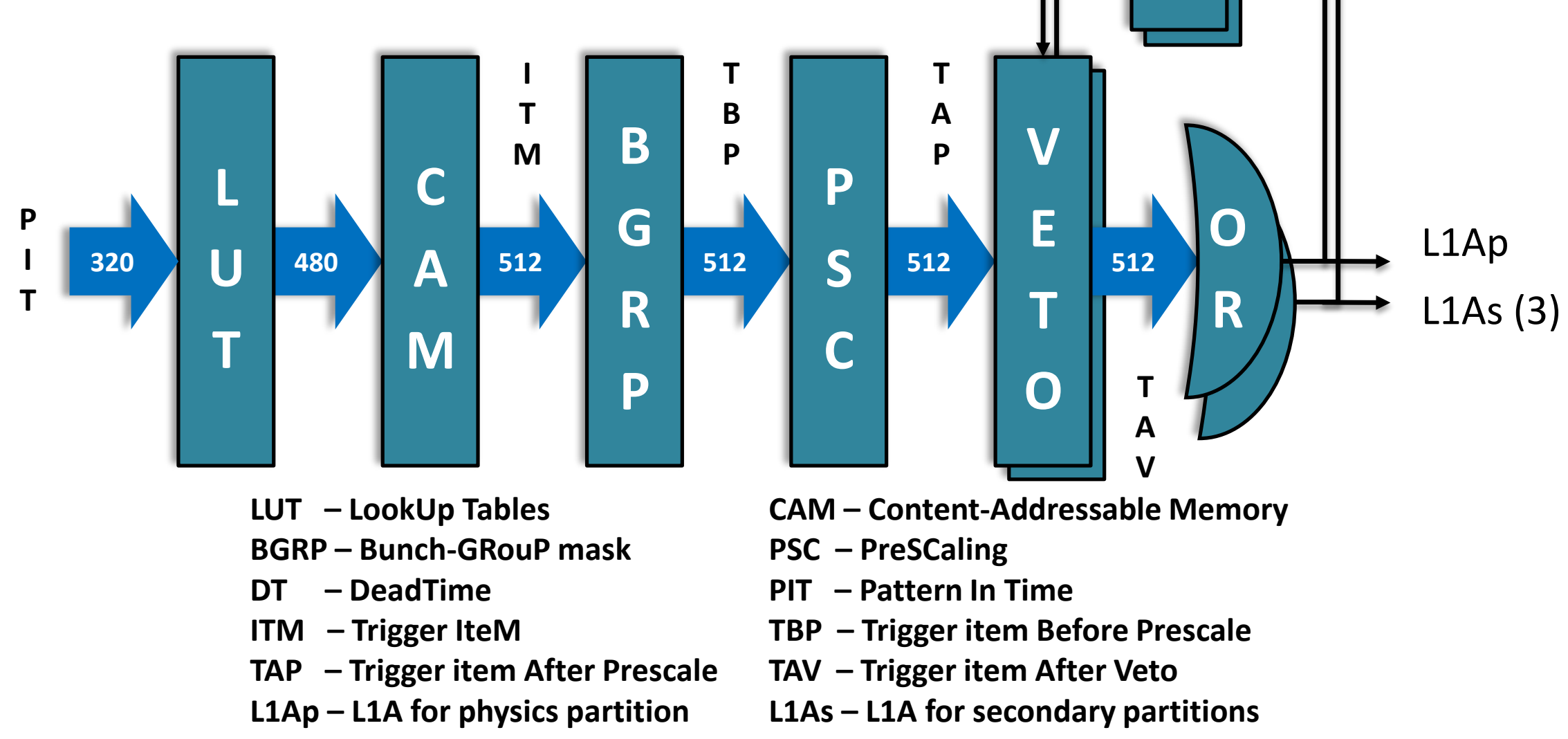




\section{CTPCORE Firmware}

- Trigger menu implemented as large CAM

- 480 data inputs, $\mathbf{5 1 2}$ match outputs

- Xilinx device architecture enables implementation of wide CAMs

- LUT configured as shift register (SRL) for data word lookup

- Carry chain for cascading CAM word

- 20 bit wide CAM in one Virtex-7 SLICEM

- 480-wide, 512 deep CAM

- 12800 SLICEs: $40 \%$ of XC7VX485T SLICEMs

- CAM content is initialized by loading shift register chain through VME

- Values are computed from the Level-1 trigger menu

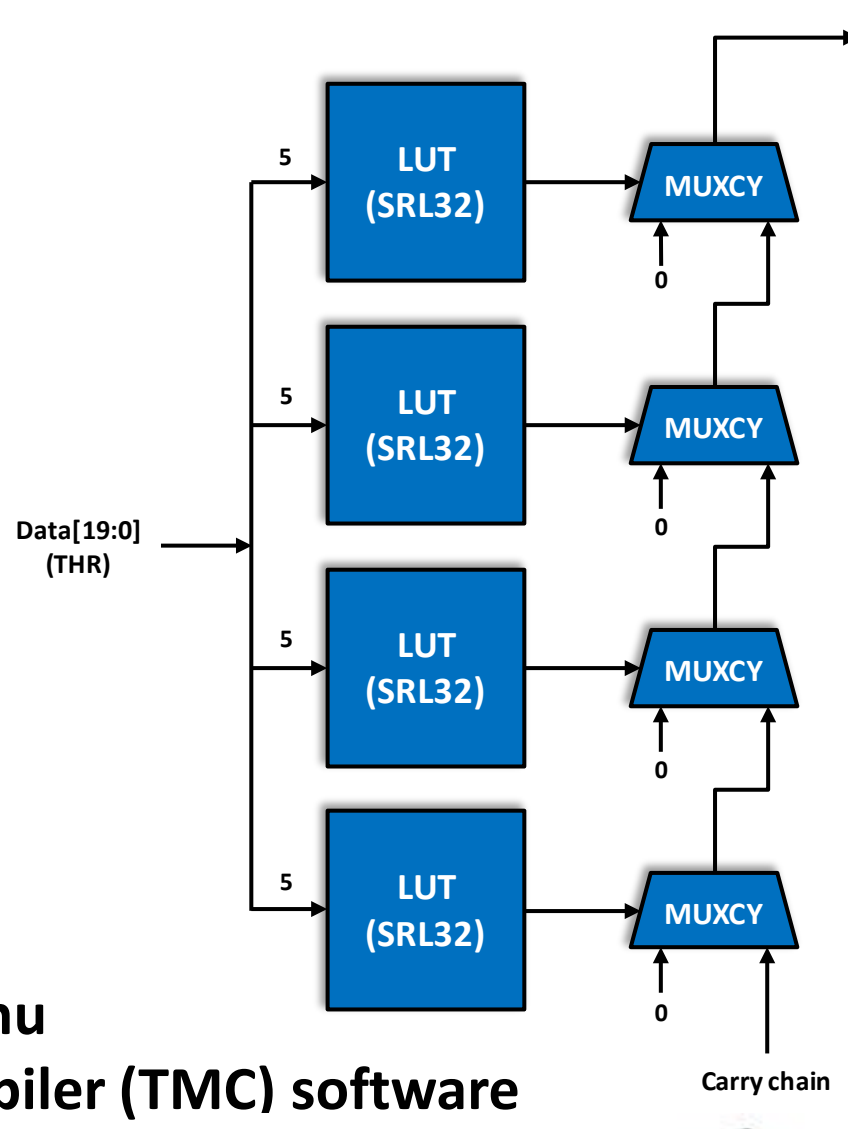

- Upgrade will require rewrite of trigger menu compiler (TMC) software

- Firmware development and prototyping

- Using Xilinx Virtex-7 development board (VC707)

- Same FPGA (XC7VX485T), different package

- Trigger path firmware $\sim \mathbf{4 0 \%}$ utilization

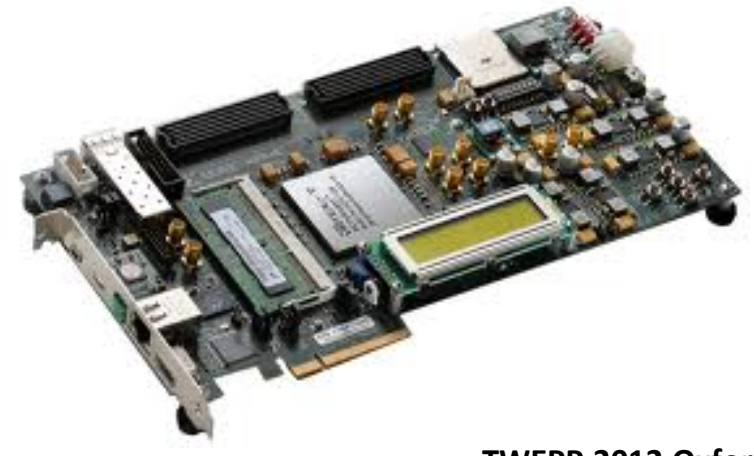




\section{CTPOUT++ Module \& Backplane}

- Upgrade CTPOUT to support more than one trigger partition

- Select trigger partition for each output cable

- Multiplex L1A \& TTYPE

- Select busy to drive

- Fan-out BC, ORB, ECR

- Multiplexing of trigger signals implemented in CPLD

- Low latency: 1/2 BC for CTPOUT

- Additional features

- Improved busy monitoring

- Programmable pattern generator for tests

- Also need to replace COM backplane to distribute signals for 3 additional trigger partitions

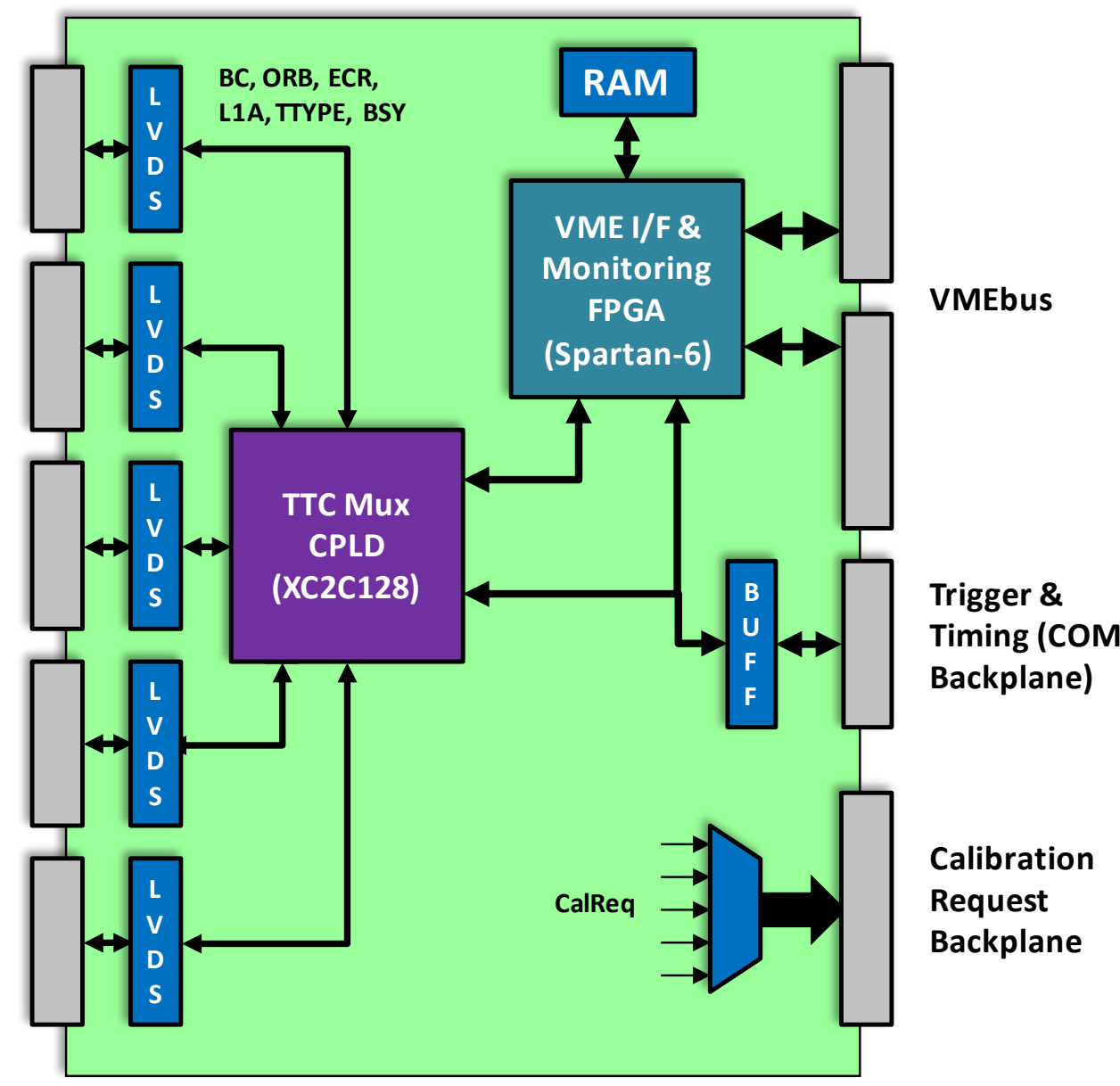
Electrical LVDS links to detector TTC partitions

- Increase the number of outputs from CTP to the TTC partitions ( $20->25$ ) 


\section{Summary}

- Phase-0 CTP upgrade to allow additional trigger inputs and to provide increased flexibility for the trigger menu

- Double the number of trigger inputs (160 $>$ 320)

- Double data rate signalling on PIT backplane

- Additional low-latency electrical inputs for topological processor

- Provision for optical inputs in addition to electrical

- Double the number of trigger items (256 -> 512)

- Add 3 secondary trigger partitions for detector commissioning and calibration runs

- Redesign several modules

- CTPCORE

- CTPOUT

- COM backplane

- Prototypes expected in Q1'13 